\title{
Surfaces
}

\section{LE CONTRÔLE DE L'IMAGINAIRE ET LA LITTÉRATURE COMPARÉE}

\section{Luiz Costa-Lima}

Volume 1, 1991

URI : https://id.erudit.org/iderudit/1065253ar

DOI : https://doi.org/10.7202/1065253ar

Aller au sommaire du numéro

Éditeur(s)

Les Presses de l’Université de Montréal

ISSN

1188-2492 (imprimé)

1200-5320 (numérique)

Découvrir la revue

Citer cet article

Costa-Lima, L. (1991). LE CONTRÔLE DE L'IMAGINAIRE ET LA LITTÉRATURE COMPARÉE. Surfaces, 1. https://doi.org/10.7202/1065253ar
Résumé de l'article

Le rôle de l'État-nation dans le processus de légitimation des études littéraires, comme mode de contrôle de l'imagination. Le prestige accordé à l'art dans le mouvement esthétique du 19e siècle, comme étant lui-même une fonction de ce contrôle, puisqu'il est basé sur la neutralisation antérieure de la force critique de l'art. La littérature comparée, l'État-nation, et l'état de la théorie. Le discours esthétique comme une tentative de contrôler la force critique de la fiction. 


\title{
LE CONTRÔLE DE L'IMAGINAIRE ET LA
}

\section{LITTÉRATURE COMPARÉE}

Luiz Costa-Lima

\section{RÉSUMÉ}

Le rôle de l'État-nation dans le processus de légitimation des études littéraires, comme mode de contrôle de l'imagination. Le prestige accordé à l'art dans le mouvement esthétique du 19e siècle, comme étant lui-même une fonction de ce contrôle, puisqu'il est basé sur la neutralisation antérieure de la force critique de l'art. La littérature comparée, l'Étatnation, et l'état de la théorie. Le discours esthétique comme une tentative de contrôler la force critique de la fiction.

\begin{abstract}
The function of the nation-state in the legitimation of literary studies as a mode of control of the imagination. The prestige accorded to art in C19th aesthetics as itself a function of this control, since it is predicated on the prior neutralisation of the critical force of art. Comparative Literature, the nation-state, and the state of theory. Aesthetic discourse as an attempt to control the critical force of fiction.
\end{abstract}


Depuis 1980, je me dédie à l'examen et à la complexification d'une certaine hypothèse: que la raison occidentale, à partir du déclin de la conception qui faisait de Dieu le garant de la vérité, s'est avérée être soupçonneuse, voire dédaigneuse, rejetant à la limite toute forme de pensée et d'expression qui ne serait pas a priori soutenue et justifiée par une méthode qu'elle justifierait, elle-même, directement. En découle, déjà pendant la Renaissance, le privilège accordé aux mathématiques, soit dans la philosophie magique d'un Cornelius Agrippa, soit dans la conception d'une science expérimentale de Galilée. En l'absence d'une indiscutable assurance transcendantale sur ce qui pourrait s'affirmer comme vérité, répondait la considération pour les mathématiques. Bien que leur raisonnement, nécessairement abstrait, puisse être gênant pour ceux qui n'étaient pas d'accord avec les prémisses platoniciennes d'un cosmos parfaitement mathématisable, cette difficulté se compensait par la force de vérification du calcul mathématique. Car, si Niklas Luhmann a bien raison de mettre l'accent sur le rôle de la perception dans la recherche d'une vérité en termes strictement humains, le manque qui, du point de vue perceptuel, affecte la démonstration mathématique est vite suppléé et dépassé par la portée d'une certitude inébranlable. Allant au-delà des percepta ${ }_{2}$ le calcul mathématique montrait que le non-<<vu $>>$ obéissait à la même régularité que le visible.

Tandis que la perception jouissait d'un privilège, l'imagination au contraire, surtout dans le cadre de la conception expérimentale de la science enfin victorieuse, se voyait ou bien méprisée ou bien entourée de normes -- les principes de l'imitatio, de la bienséance et de la vraisemblance -- que la poétique de la Renaissance jugeait capables de bien orner ses produits pour le commerce des gens illustres. De ce fait, plus un genre était légitimé, plus s'exerçait sur lui le contrôle ou plus grand était le besoin de lui concéder une marge de tolérance (la tolérance ne peut être pensée sans l'existence de son contraire, l'interdiction).

C'est à partir de ces prémisses que se dresse ce que j'appelle le contrôle de l'imaginaire. (Je ne tiens pas compte ici des arguments historiques qui ont aidé à le promouvoir, comme l'exemple par excellence des luttes religieuses; ni d'un facteur socio- historique non moins important: la reconnaissance progressive de la subjectivité en tant qu'individuelle).

Bien que les bases de ce contrôle se font déjà fait sentir dans la théorie platonicienne des idées et dans la conception épistémologique d'Aristote, ou, plus largement, depuis que la conception de la vérité conçue comme antérieure à son énonciation a vaincu la conception opposée des sophistes, j'ai préféré ne pas recouvrir un terrain aussi considérable. Je ne suis pas un helléniste et même si j'en étais un, le choix d'une telle étendue temporelle empêcherait préalablement l'avancement significatif de cette hypothèse. J'ai donc pris le parti d'étudier, sous cet angle précis, la pensée moderne. 
Le premier objet de mes questions a donc été la poétique de la Renaissance. Comme leurs résultats fondamentaux se trouvent dans un livre qui a déjà été traduit en anglais et en allemand, je n'ai pas jugé utile de reprendre ici les conclusions auxquelles ce travail a mené. J'aimerais mieux souligner le pas qui s'en est suivi, dans les deux livres qui achèvent de constituer la trilogie du Contrôle, Sociedade e discurso ficcional (1986) et $O$ Fingidor e o censor_(1988).

Lors d'une première recherche, il s'est avéré exister une différenciation entre des formes de contrôle. J'ai donc commencé à parler d'un contrôle à dominante religieuse, exercé au cours des XVIe et XVIIe siècles, et d'un autre, à dominante progressivement laïque et scientifique, en voie d'expansion depuis le Siècle des Lumières en France. En plus, d'un point de vue purement heuristique, j'ai proposé l'existence de trois cercles: le cercle proprement européen, le cercle hispano-américain et le cercle brésilien. Chacun d'eux a été traité ou bien de façon globale et non minutieuse, ou bien au moyen de cas particuliers. Je n'ai pas écrit et je n'écrirai pas une histoire systématique du contrôle de l'imaginaire, même si je ne m'y intéressais qu'en tant que voie royale (c'est-à-dire celle qui se constitue de fictions verbalement réalisées), parce qu'une tâche pareille ne pourrait être menée à terme par une seule personne vivant en plus dans les conditions précaires du tiers-monde. Je serai heureux si cette hypothèse est jugée valable dans le sens d'une meilleure compréhension de l'oeuvre fictionnelle dans notre monde.Comme Sociedade e discurso ficcional et $O$ Fingidor $e$ o censor doivent paraître prochainement en anglais, en versions abrégées, je crois pouvoir m'attendre à prendre part au dialogue de la communauté internationale des confrères.

Il me faut maintenant considérer quelle conception de la littérature, et surtout quelle conception de la littérature comparée est comprise par cette démarche. Dans quelle mesure, faut-il se demander tout d'abord, ce type d'interrogation ne mène-t-il pas à confondre encore la littérature et l'histoire des idées? La littérature ne serait-elle qu'une de ses manifestations?

Cette question n'est pas entièrement dépourvue de sens. L'hypothèse avec laquelle je travaille s'oppose tout autant à l'isolement de la série littéraire qu'à la prétention que ses composantes se trouvent investies de traits textuels qui lui seraient propres, c'est-à-dire à l'existence d'une littérarité textuelle et définitivement établie. Ou encore, qu'un produit une fois modelé par des procédés littéraires tout en pouvant tomber en discrédit ou, au contraire, acquérir une renommée auparavant inconnue, resterait pour toujours identifié comme étant un produit littéraire. Une telle caractérisation de la littérature s'est amorcée au début de notre siècle, soit par le biais de Croce, soit par le travail des formalistes russes, comme réaction à l'historicisme positiviste du XIXe siècle; elle s'est propagée avec $<<$ l'explication de texte $>>$ des Français et le New Criticism anglo-saxon pour atteindre son apogée avec le courant que l'on a dénommé le 
structuralisme français. Si l'accent que l'on mettait alors sur les procédés constitutifs de l'oeuvre a dignifié l'objet littéraire, et si les microanalyses qu'on en faisait l'ont libéré de ce caractère de fantôme murmurant dans le cimetière de l'histoire, en revanche l'identification des éléments psychosociaux aux facteurs <<extrinsèques $>>$ (Wellek) a, d'une part, stérilisé ce qu'il y avait de plus fécond dans les investigations de Tynianov et de Mukarovsky et, d'autre part, contribué à déconnecter l'oeuvre littéraire du sol à partir duquel elle prend son sens: le sol historique. Reconnaissant leur importance, mais divergeant de la conception qui prend l'oeuvre littéraire, surtout son espèce poématique, pour le résultat de la combinaison de traits textuels, je suis évidemment amené à remettre en question la tradition des études littéraires.

Pour ne pas m'égarer dans un océan de courants, de noms et de distinctions, il vaut mieux envisager la question selon la tradition qui, depuis les premières décennies du siècle dernier, l'a emporté sur toutes les autres.

Personne n'ignore que l'idée d'État-nation est à la base de l'institutionnalisation de l'étude de la littérature comme un corps autonome et non plus confondu avec les formes de l'éloquence. Les littératures se distinguent alors selon le principe de la nationalité, et les auteurs se trouvent disposés en couches diachroniques qui maintiendraient et renouvelleraient un prétendu esprit national. Outre cette disposition historique, l'objet littéraire était encore soumis à l'examen philologique. D'où l'importance que prennent les catégories de source, d'influence, de causalité, de vicissitudes biographiques, de détermination de variantes et de fixation du texte considéré comme critique et définitif (Cf. Bernard Cerquiglini: 1989). Justifiées de loin par l'idée du Volksgeist, les études littéraires du siècle dernier présentaient la curieuse particularité de ne pas se poser de questions sur la nature de leur propre objet. Tout pourrait les concerner sauf, précisément, pourquoi ceci ou cela appartenait à la littérature. D'où l'aisance avec laquelle on pratiquait l'anachronisme, identifiant comme étant littéraires des oeuvres qui, dans les siècles précédents, relevaient du concept ultérieur de littérature (Cf. Marc Fumaroli: 1980). L'objet de cette reprise est assez connu pour que l'on s'y attarde encore. Il faut pourtant signaler que la légitimation des études littéraires par le principe de nationalité impliquait la victoire d'une des tendances qui pouvaient s'observer dans la réflexion romantique sur la littérature. Je la formule au passage. Si l'on repense les essais de Schiller, Über die ästhetische Erziehung des Menschen, de Shelley, A Defense of Poetry, et de Coleridge, <<On Poesy or art $>>$, on pourra vérifier que l'un de leurs communs dénominateurs tournait autour de la fonction sociale que l'art serait en mesure de remplir. Chez Coleridge, l'art est le médiateur et, de ce fait, le réconciliateur de l'homme avec la nature. Il aurait donc pour fonction de rétablir l'équilibre qu'avait défait le développement de la société. 
Cette réconciliation était en plus destinée à ces peuples qui avaient dépassé ce stade inférieur où les sons ne sont que $<<$ a mere expression of passion>>. C'est pourquoi l'auteur observait:

The so called music of savage tribes as little deserves the name of art for the understanding, as the ear warrants it for music (Coleridge, S.T.: 1818, 253).

Avant même de me poser des questions sur les conséquences de l'évolutionnisme dans la pensée de Coleridge, je me demande ce que peut signifier l'équilibre déclaré que l'art était censé rétablir entre l'homme et la nature. Le fait de le voir d'emblée comme la justification de la supériorité et de la maîtrise des nations européennes sur les tribus sauvages serait tout simplement prendre un chemin commode. Mais le rapprochement avec Schiller nous permettra d'arriver à un meilleur résultat. Dans l'impossibilité de procéder à l'examen adéquat, je souligne d'ores et déjà chez le poète allemand une plus grande netteté à l'égard d'un diagnostic de la modernité. Celle-ci se compose, selon l'essai de 1795, d'individus fragmentés. D'où, simultanément, la supériorité de l'homme grec et la fonction dont devait s'investir l'art du temps:

Pourquoi chaque Grec se qualifiait-il de représentant de son temps et pourquoi l'homme moderne ne peut-il oser le faire? Parce que celui-là recevait ses formes de la nature, qui unit tout, et que celui-ci les reçoit de l'entendement qui sépare tout (Schiller, F.: 1795, lettre 6, 19).

Si l'homme grec, à la différence de l'homme moderne, se tenait en contact avec la nature, la fonction actuelle de l'art serait, bien entendu, la réintroduction de cet équilibre perdu. Voilà le rôle de l'éducation esthétique proposée comme le seul moyen, aux yeux de Schiller, capable de convertir le rapport non satisfaisant de l'individu à l'État. Mais comment y arriver? J'en suivrai uniquement le sillon principal. L'éloge schillerien de l'homme grec m'amènerait à faire fausse route si l'on arrivait à la conclusion que la position de Schiller n'était que nostalgique. Loin de là: il dira expressément que les questions posées au passé nous montrent toujours <<que le goût et la liberté s'évitent mutuellement et que la beauté ne fonde son domaine que sur le déclin des vertus héroïques >>. (Idem, lettre 10,40). Cette insatisfaction de l'exemple du passé se fait plus concrète quand on relit le passage qui suit:

Que d'entendements débiles ne se sont mis en discorde par rapport à l'ordre social parce que la fantaisie du poète a bien voulu ériger un monde où tout se passe autrement(...), Ibid., lettre 10, 39). 
Ce qui veut dire que l'éducation esthétique visée ne se confond absolument pas avec la stimulation de l'esprit critique, ce dernier étant confondu avec le délire de la fantaisie et l'esprit de discorde. L'éducation esthétique serait mise en oeuvre par l'harmonisation de l'impulsion sensible -- où l'individu est gouverné par ses appétits -- à l'impulsion formelle - -- où, au contraire, aucune dépendance par rapport à l'objet ne vient entraver la liberté d'action du sujet. C'est par cette conciliation que, dans l'État esthétique, l'artiste harmoniserait le domaine du scientifique et celui de la moralité. La fonction de l'art se trouverait en rapport direct avec la formation de belles âmes sages. La loi internalisée, le citoyen serait en mesure d'avoir une pleine liberté d'action. Cet attardement sur le texte schillerien m'a permis: (a) de mieux voir la direction esthétique comme l'une des forces centrales de justification de l'art dans la modernité; (b) grâce au parallèle avec l'essai de Coleridge, de mieux comprendre quel était le chaînon liant la justification esthétique au principe de nationalité. Au plus clair: le rôle de réconciliateur de l'homme et de la nature affecté à l'art ne pourrait s'accomplir que dans les nations civilisées; c'est-à-dire, selon les mots de Coleridge, celles où les sons ne sont plus la simple expression des passions ou, selon les mots de Schiller, celles qui se constituent de citoyens ayant appris à combiner leurs impulsions sensibles et formelles. Ce chaînon ne se fait pas voir parmi les louanges d'habitude réservées à la littérature. Cependant, quand il aura été saisi, comment ne pas le reconnaître dans l'indication que donnait Curtius du modèle du bon Européen:

Ich weiss nur eine Art ein guter Europaer zu sein Macht die Seele seiner Nation haben, und sie mit Macht nahren von allem, was es Einzigartiges gibt in der Seele der anderen Nationen, der befreundeten oder der feindlichen (Je ne connais qu'une façon d'être un bon Européen: c'est d'avoir avec force l'âme de sa nation et, avec force, de la nourrir de tout ce qu'il y a de singulier dans l'âme des autres nations, amies ou ennemies (apud Wellek, R.: 1963, 288)).

Le citoyen civilisé se caractériserait par l'internalisation de l'âme nationale sans pour autant devenir un chauviniste. Le journal parisien d'Ernst Junger est peut-être la meilleure démonstration de comment un nazi pouvait tenir dans ce moule. On peut donc dire que la conception de la littérature qui était à la base de la légitimation de son étude universitaire impliquait une vision esthétisante de la part de son usager -- esthétisante parce que noncritique, mise au service d'une conception impériale des nations $<<$ civilisées $>>$. Ou, pour parler de façon plus directe: elle était au service de la conception impériale de l'Occident.

Rien de neuf si l'on y ajoute que l'idée de la littérature comparée était et a été directement liée à la conception de la littérature nationale. Si elle était réduite, comme le souligne un comparatiste traditionnel, à une place marginale par $<<$ the chauvinism and provincialism of the other literary departments >> (Levin, H.: 1969, 82), son évidence ne supposait pas moins 
pour autant le rapport entre au moins deux nationalités différentes dont l'une était identifiée à la source rayonnante et l'autre au domaine qui en bénéficierait.

Puisque c'est dans ces termes que je conçois et formule la vision traditionnelle et majoritaire de la littérature comparée, il est évident que nos rapports ne vont pas dans le sens de l'affinité. Nier cette vision, c'est repousser la vision esthétique de la littérature, mépriser le critère national dans lequel elle est ancrée, renier le positivisme événementiel et antithéorique qui est à sa base.

Jusqu'à présent, la démarche adoptée pour cet exposé a consisté à mettre l'accent sur les courants dont s'écarte ma propre orientation théoricoanalytique. Il s'agit maintenant d'inverser la direction prise et de signaler la lignée avec laquelle elle a des points de contact, d'une manière ou d'une autre. Dans l'article $<<$ Comparative literature and literary theory $>>$, Jonathan Culler souligne l'ambiguïté qui gouverne les rapports entre la littérature comparée et la théorie de la littérature. Le comparatiste, en bénéficiant de la difficulté contre laquelle butent ses confrères pour justifier théoriquement la notion d'unité d'une littérature nationale, a sur ces derniers l'avantage d'un certain support théorique qui le justifie académiquement:

Students of Comparative Literature have always invested time and ingenuity in arguing that genres, or periods or period styles, or themes, were appropriate objects of literary study and ought to take precedence over that other traditional object, the literature of a nation state organized in its chronological sequence (Culler, J.: 1979, 170-1).

Mais c'est précisément à ce moment-là que l'ambiguïté se révèle: malgré la $<<$ vocation $>>$ théorisante du comparatiste, Culler observe à juste titre qu'il ne connaît aucun département de littérature comparée où ce programme soit rigoureusement mis en oeuvre. Cela s'explique, dit-il, par le lien ombilical attachant les départements de littérature comparée et de littératures nationales:

The point is that any theoretical argument which puts in question the importance of national boundaries and promotes some other sort of unit, (...) 
will suggest, if rigorously developed, that organization in terms of national literatures should yield to a theoretically grounded poetics of some kind (Culler, J.: Op.cit., 171).

On pourrait croire qu'il se passe ici quelque chose qui ressemble, aux yeux de Hegel, à l'alliance entre les seigneurs et les esclaves: la faim du loup est assouvie par la docilité des agneaux. Que je sache, cette dénonciation de Culler n'a pas suscité de remous. En effet, le principe de la nationalité, enraciné, comme on l'a vu, dans la justification esthétique de l'art et de la littérature, reste extrêmement puissant. Il en découle la $<<$ crise prolongée de la littérature comparée >> (Wellek, R. 1963, 290). Et si on n'en parle pas assez, c'est peut-être tout simplement parce qu'ils sont peu nombreux, ceux qui auraient intérêt à en discuter. Je n'aurais pas la naïveté de croire que la simple référence à un état de choses assez connu représente un pas accompli. Mon objectif est bien plus modeste: la situation de manque que l'on vient de signaler peut être tenue pour un des effets de la défaite d'une autre ligne de force qui s'était révélée dans la réflexion romantique. On avait remarqué ci-avant le lien existant entre la proposition de $<<$ fonctionnalisation $>>$ de l'art par le relief de l'<<esthétisation $>>$ et le critère, enfin vainqueur, des littératures nationales. Il faudrait donc souligner maintenant la corrélation opposée qui s'établit entre la recherche d'une caractérisation du poétique comme activité critique et l'absence d'une réflexion théorique, demandée depuis des décennies. De même que, pour la première relation, le recours à Asthetische Erziehung a été décisif, pour la seconde, il faudrait faire appel aux deux collections de fragments de Friedrich Schlegel, les <<Kritische Fragmente>> (179?) et les Athenaeum Fragmente (1798). Ne pouvant les exposer ici, je me limiterai à souligner un de leurs aspects fondamentaux: celui de la relation entre la poésie, la critique et l'esthétique.

Pour ce qui est de la relation entre la critique et la poésie, les fragments 117 et 57 des KF sont essentiels. Du premier, on peut se contenter de retenir l'ouverture: <<Poesie kann nur durch Poesie kritisiert werden>> (La poésie ne peut être critiquée que par la poésie). Mais on ne peut en aucun cas faire l'économie du second.

Si certains amants mystiques de l'art, pour lesquels chaque critique équivaut à une dissection et chaque dissection à la destruction du plaisir, étaient conséquents, le meilleur jugement sur la meilleure des oeuvres serait: quelle merveille. Il y a bien des critiques qui ne disent rien de mieux; seulement, avec plus de mots.

Ce n'est qu'en apparence que ces deux fragments sont contradictoires. Tous les deux soulignent que la critique ne peut pas être une activité extérieure au poétique lui-même (ce qui ne se confond pas avec l'affirmation qui 
prêcherait qu'elle doive être un poème!); qu'elle est tout aussi poïesis que le poème lui-même. C'est la raison pour laquelle elle ne se confond pas non plus avec les exclamations enthousiastes. Bref, pour Schlegel, critique et poésie forment des parties inséparables; ce que le jeune Benjamin de la dissertation sur la critique romantique avait étonnamment bien saisi:

Ce n'est qu'avec les romantiques que l'expression $<<$ critique d'art $>>$ s'est opposée en définitive à celle qui lui était antérieure: $<<$ juge d'art $>>$. (Benjamin, W.: 1919, 52).

Or, cette emphase de la critique en tant qu'activité intérieure, $<<$ kantienne $>>$, puisqu'orientée par l'exploration et la vérification des limites d'un mode de connaissance, et non pas extérieure -- ce qui est propre aux juges qui prononcent des sentences --, Schlegel l'écartait de l'esthétique. Comme on le voit dans le fragment 40:

Dans le sens qui lui a été attribué et qui est courant en Allemagne, le mot 'esthétique' trahit, on le sait, d'un seul coup, la pleine ignorance et de la chose désignée et de la langue avec laquelle on la désigne. Pourquoi est-il maintenu? (Schlegel, F.: Op.cit., 170))

Le succès qu'obtiendra l'essai de Schiller montre que l'erreur qui était à l'origine du choix du mot $<<$ Asthetik $>>$ répondait à un besoin historique. La culture bourgeoise met l'art <<au-dessus de tout soupçon $>>$ pourvu qu'il s'investisse d'un instrument qui le neutralise. Éloignée de sa fonction polémique, comme le montrerait l'écoulement du temps, la fiction se fait divertissement ludique, moyen éventuel de remplir l'oisiveté des bourreaux $<<$ civilisés >> . J'insisterai simplement sur le point suivant: le contrôle de l'imaginaire que je cherche à souligner n'est pas démenti par le prestige qu'accordera à l'art le XIXe siècle. Ce prestige était ancré dans la neutralisation préalable de son potentiel polémique.

\section{[[section]]}

Voilà l'archéologie que je pourrais tracer de ma position. De la tradition constituée à partir de la fin du XVIIIe siècle, je m'écarte du versant esthétisant, nationaliste et, par la suite, événementiel-positiviste. Je me rapproche, par contre, du versant critique, internationaliste et interprétatif. 
Bien que quasi-télégraphique, cet examen ne peut se terminer avant d'en arriver à la scène actuelle. Signalant d'abord la discordance par rapport à la tentative de caractériser la littérature au moyen de traits textuels propres, je rendais d'une certaine façon ma tâche plus facile: dans le marché académique, les New Critics et les méthodes strictement structuralistes sont en baisse. Mais que dire du déconstructionnisme? (Inutile de se plaindre encore du manque d'espace. L'examen devra être court). Je me borne à en détacher la donnée indispensable: la réflexion sur les frontières de la littérature.

Ils sont nombreux les mérites des déconstructionnistes. Ils ont levé le cordon sanitaire dont les New Critics avaient entouré la poésie, lui interdisant le contact avec le monde des idées. L'un d'eux, Paul de Man, dans l'article <<Aesthetic formalization: Kleist's Über das Marionettentheater $>>$, a attiré notre attention sur la force et les conséquences de la justification esthétisante de l'art. Ce sont eux qui ont poussé le spécialiste en littérature à se réintéresser à des textes académiquement éloignés de son domaine, surtout des textes philosophiques ou religieux. Ce sont eux aussi qui ont fait éclater l'a priori de l'unicité de l'oeuvre en poursuivant la dénonciation de l'aura enveloppant le $<<$ créateur $>>$. À tout cela vient s'ajouter la dignité qu'ils ont octroyée au travail portant sur la littérature capable maintenant d'influer sur les recherches des anthropologues et des historiens. Leur influence peut se mesurer à la dissémination de leurs idées dans un livre comme The Genesis of Secrecy dont l'auteur, Frank Kermode, ne se prend certes pas pour un adepte de ce courant.

Plus généralement, il faudrait louer le combat qu'ils livrent contre les classements clos et étanches qui établissent la place $<<$ définitive $>>$ de ce qu'il faudrait nommer religion, science, philosophie, critique, fiction, etc. Je crois même qu'un livre comme Criticism in the Wilderness de G. Hartman est aujourd'hui une lecture indispensable pour ceux qui ne se contentent pas de considérer la critique comme un commentaire, une mise en ordre systématique ou une divulgation de l'oeuvre littéraire. Tout aussi bien que la poésie, l'activité critique est un problème de langage. La critique n'aura qu'une importance éphémère si elle n'est pas animée d'une <<impulsion fictionnelle $>>$ ou si elle ne rend pas son lecteur / pp. 15-16/ conscient des fictions critiques contenues dans les textes dont elle traite (Cf. Hartman, G.: 1980, 201). Elle ne se place donc ni en-deçà ni au-delà du terrain où travaille le poète. L'acte de déconstruction implique la démystification du génie créateur, de l'originalité absolue et du privilège conféré à la notion d'auteur. Par contre, il suppose <<the awareness that all writing is a fusion of heterogenous stories >> (Hartman, G.: 1986, 13). Les murs sont éliminés qui enfermaient dans des compartiments étanches poètes et penseurs, romanciers et historiens, narrateurs et scientifiques. 
Tout cela est fécond et stimulant. Mais l'idée du texte, comme une sédimentation multiple et hétérogène qui implique des discordances et des contradictions internes, signifierait-elle que les classifications des textes ne sont que tributaires du mythe du sujet individuel autonome et créateur? Telle est, semble-t-il, l'orientation des meilleurs déconstructionnistes. Ainsi, par exemple, dans son essai sur l'épisode de la lutte entre Jacob et l'ange (Gen.,32), Hartman finit par affirmer:

The universality of Jacob's combat with the angel lies, finally, in that struggle for a text -- for a supreme fiction or authoritative account stripped of inessentials, of all diversions, of everything we might describe as arbitrary, parochial, even aesthetic (Hartman, G.: 1986,16).

La position qu'adopte l'auteur dans ce passage se fait plus claire, quant à ses conséquences, lorsqu'on la met en relation avec la réflexion de Paul de Man dans The Resistance to theory. En la définissant comme une résistance à la lecture elle-même, de Man y inclut Jauss et Iser parce qu'ils $<<$ do not allow for the problematization of the phenomenalism of reading and therefore remain uncritically confined within a theory of literature rooted in aesthetics $>>$ (Man, P. de: 1986, 18). À cette lecture donc, s'oppose une autre d'ordre rhétorique, c'est-à-dire qui s'adresse à la détermination des stratégies expressives présidant au texte analysé. Les lectures rhétoriques, ajoute-t-il, <<are theory and not theory at the same time, the universal theory of the impossibility of theory. [...] Nothing can overcome the resistance to theory since theory is itself this resistance (Man, P. de: Op.cit., 19).

Il est impossible de rendre ici pleine justice à la force de ce passage. Je me contenterai de faire l'observation suivante: la lecture rhétorique incorporerait la résistance à la théorie elle-même, c'est-à-dire qu'elle rendrait cette résistance -- qui ne se confond certes pas avec la résistance banale, fondée sur les clichés de la participation intuitive, de l'émotion provoquée par la poésie, de l'intention de l'auteur, etc. -- positive parce que la rhétorique dévoilerait le texte comme étant un ensemble indéterminé, tendant toujours à se disséminer dans d'autres figures, dans d'autres interprétations, dans d'autres...textes.

Comme l'avance Paul de Man, la proposition déconstructionniste a ce défaut qu'elle n'envisage pas le rendement du texte au sein des sociétés dans lesquelles il circule. Dans ce sens, le déconstructionnisme prend la même route qu'avait empruntée le New Criticism: l'un et l'autre hypostasient le texte. Si les New Critics se bornaient au texte littéraire, les déconstructionnistes élargissent leur rayon d'action pour parvenir au travail même du langage. Il est sans doute utile et fécond de déconstruire l'opposition entre littérarité et langage figuré, et de montrer, comme le dit 
Derrida dans <<La Mythologie Blanche $>>$ (Marges de la philosophie), le saut métaphorique au moyen duquel Aristote caractérisait la métaphore ellemême. Celle-ci n'est donc plus spécifique aux discours ornementaux, et sa présence dans le discours scientifique le plus dur nous contraint à renoncer à l'idée de la science comme la reconnaissance de propriétés déjà contenues dans la chose per se. J'admets volontiers l'instabilité constitutive du texte; j'admets également que tout récit, peu importe s'il est en prose ou en vers, est bien une construction et, par conséquent, que la fiction n'a plus de frontière fixe. Mais ne détacher que cet aspect signifie professer encore une espèce de substantialisme: la substance du langage serait son indétermination même; de là sa prégnance disséminative. Or, tout texte présuppose un langage, et le langage se trouve toujours dans une situation de jeu-de-langage, c'est-à-dire qu'il suppose toujours des partenaires qui le reçoivent, l'élaborent et s'en servent suivant des normes et des conventions sociales. S'en écarter au nom de l'indétermination constitutive du langage serait le rendre, littéralement, un objet métaphysique. Il faudrait donc ajouter que la détermination du sens et du choix, parmi les diverses possibilités de signification qu'offre un texte, varient selon le réseau qui préside à sa production et à sa réception. Il n'y a certes pas de traits textuels distinguant un texte philosophique d'un texte poétique ou un texte poétique d'un texte religieux; mais à l'identification de leurs procédés rhétoriques, il faudrait ajouter les questions historico-sociales posées en rapport avec les situations discursives où ces procédés ont lieu. Plus clairement, disons qu'à l'indétermination du langage, il faut ajouter l'analyse de l'idée de discours: du discours compris comme une forme de territorialité. Ce qui veut dire qu'il se constitue d'une série de marques, verbales et/ou sémiologiques, signalant au partenaire singulier ou pluriel quelle est la forme de réception attendue. Historiquement, les textes glissent d'une forme discursive à une autre dans la mesure où ils ont la possibilité d'actualiser différentes constellations de traits, qui se reconnaissent comme des marques propres à un autre discours qui n'est plus celui qui était visé originairement. L'horizon esthétique, et sur ce point je suis d'accord avec Paul de Man, établissait une limite arbitraire à la mouvance du texte. L'horizon rhétorique, en revanche, accentue à tel point l'indétermination du texte qu'il rend et son fonctionnement et ses différentiations impossibles à reconnaître, socialement parlant.

Encore quelques mots. S'il n'y a pas de marques propres à la littérature, elle n'en a pas moins -- depuis le Quichotte, au moins -- un territoire qui lui est propre: celui du fictionnel. Dans l'impossibilité de l'analyser, ajoutons simplement que le fictionnel est, non pas ce qui s'exempte de la dichotomie 'faux ou vrai', mais ce qui permet la mise en perspective de la vérité, c'est-àdire sa remise en question. Son caractère sui generis consiste, comme l'a bien montré $\mathrm{W}$. Iser, à stimuler cette mise en question sans proposer luimême une autre vérité. Quand, par conséquent, je parle du contrôle de l'imaginaire, je me pose des questions sur les conditions historico-sociales qui empêchent ou qui restreignent l'actualisation du territoire de la fiction. Il est pourtant facile de comprendre la raison de sa présence puisque dans une société guidée par un principe de vérité expérimentale, telle que l'est la société de la modernité occidentale, l'existence d'un territoire par trop 
$<<$ questionneur $>>$ de la vérité et qui, en plus, ne propose pas une autre vérité, ne pourrait que faire scandale. La justification esthétique de la littérature et de l'art a donc cherché à l'apprivoiser. C'est pourquoi je la considère comme un exemple puissant du bien-fondé de notre hypothèse.

\section{Universidade do Estado de Rio de Janeiro}

\section{Brésil}

Surface Page d'Acceuil/Home Page

\section{Références bibliographiques}

Benjamin, W.: <<Der Begriff der Kunstkritik in der deutschen Romantik >>, in Gesammelte Schriften, vol. I - 1, R. Tiede man et H. Schweppenhauser (édit.), Frankfurt a.M ., Suhrkamp Verlag, 1980.

Cerquiglini, B.: Éloge de la variante. Histoire critique de la philologie, Paris,Seuil, 1989.

Coleridge, S.T.: $<<$ On poesy or art $>>$, in Biographia literaria, v.II, J.Shawcross (édit.), London, Oxford university Press, 1958.

Culler, J. : $<<$ Comparative literature and literary theory $>>$ in Michigan germanic studies, 5, 2, 1979.

Fumaroli, M. : L'Âge de l'éloquence. Rhétorique et $<<$ res literaria $>>$ de la renaissance au seuil de l'époque classique, Genève, Droz, 1980.

Hartman, G. : Criticism in the wilderness. The study of literature today, New Haven London, Yale University Press, 1980.

Kearney, R. : The Wake of imagination, Minneapolis, University of Minnesota Press, 1988.

Levin, H. : <<Grounds for comparison $>>$, repris in Grounds for comparison, Cambridge (Mass.), Harvard University Press, 1972.

Luhmanrl, N. : Macht, Stuttgart, Ferdinand Enke Verlag, 1975. 
Man, P. de : The Resistance to theory, Minneapolis, University of Minnesota Press, 1986

Schiller, F. : Über die ästhetische Erziehung des Menschen, Stuttgart, Reclam, 1965.

Schlegel, F. : $<<$ Kritische Fragmente $>>$, in Werke in zwei Bande, vol. I, Berlin - Weimar, AufbauVerlag, 1980.

Strauss, L. : Perscution and the art of writing, Chicago-London, The University of Chicago Press, 1988.

Wellek, R.. : <<The Crisis of comparative literature $>>$, in Concepts of criticism, New Haven-London, Yale University Press, 1964. 